\title{
Anaesthesia Management for Ablation Therapy in Post Heart Transplant Arrhythmia*
}

\author{
Sunisa Prapaitrakool, Daniel Tom Bainbridge, Ronit Lavi ${ }^{\#}$ \\ Department of Anaesthesia and Perioperative Medicine, London Health Science Centre, University of Western Ontario, London, \\ Canada. \\ Email: sprapait@uwo.ca, Daniel.Bainbridge@lhsc.on.ca, "Ronit.Lavi@lhsc.on.ca
}

Received April 30 ${ }^{\text {th }}, 2013$; revised June $1^{\text {st }}$, 2013; accepted July $15^{\text {th }}, 2013$

Copyright (c) 2013 Sunisa Prapaitrakool et al. This is an open access article distributed under the Creative Commons Attribution License, which permits unrestricted use, distribution, and reproduction in any medium, provided the original work is properly cited.

\begin{abstract}
In this case series we describe the anaesthetic management of atrial ablation for arrhythmia in three post heart transplant patients. These patients provide a unique challenge to the anaesthesiologist, as heart physiology, end organs effects, procedural related factors should all be part of the specific anaesthetic plan tailored for each patient individually. The different anaesthetic techniques applied, and procedure related complications are evaluated. Anaesthetic management, procedural related difficulties, pharmacologic aspects, and possible associated complications are reviewed.
\end{abstract}

Keywords: Ablation; Heart Transplant; Arrhythmia

\section{Introduction}

Heart transplantation has become a therapeutic option for a variety of end-stage heart diseases with over 2000 cases performed annually in the United States and approximately 3500 cases worldwide [1]. As a result of an increasing number of patients surviving cardiac transplantation and the relatively high incidence of atrial arrhythmias in these patients, ablation procedures under anaesthesia may be required $[2,3]$. Percutaneous catheter ablation is done with applying electrical triggers around the pulmonary vein ostia in order to accomplish pulmonary vein isolation using radiofrequency current. Radiofrequency ablation applies high-frequency, low-voltage electrical energy to the endocardium via a catheter-based electrode that leads to resistive thermal injury and subsequent coagulative necrosis of the tissue, thereby blocking reentrant electrical circuits causing atrial fibrillation [4]. Other reports have focused on the mechanism for atrial flutter post heart transplantation and potential therapy from cardiology perspective [5,6]. In this report we will focus on Anaesthesia management for ablation therapy in post heart transplant arrhythmia.

The transplanted heart physiology is different, and can complicate anaesthetic management. Therefore, anaesthesiologists should be aware of particular problems and

\footnotetext{
*The authors declare no financial support or conflict of interest.

\#Corresponding author.
}

risks associated with ablation procedures in this unique group of patients. We present three cases of atrial ablation due to arrhythmia after heart transplantation and discuss the anaesthetic considerations for these procedures.

\section{Case Report}

Written informed consent was obtained from all patients.

\subsection{Case \#1}

A 47-year-old man received a cardiac transplantation due to sarcoid cardiomyopathy 6 years before the ablation procedure with a biatrial technique. He presented with recurrent episodes of atrial fibrillation refractory to both medical treatment and multiple cardioversion attempts. He developed renal compromise with a slightly increase in creatinine after the transplant. His current immunosuppressants were cyclosporine and mycophenolate mofetil. Electrophysiologic (EP) studies and radiofrequency ablation were done twice in the last 2 years.

The first EP study showed supraventricular tachycardia (SVT) from Wolff-Parkinson-White syndrome. The patient received sedation with both a propofol and fentanyl infusion which was well tolerated for the first 90 minutes. It was then converted to a general anaesthetia using a laryngeal mask airway, and sevoflurane to provide adequate level of anaesthesia for the next 100 min- 
utes of the procedure.

The second EP study revealed atrial fibrillation originating from the recipients' atrial cuff. Conscious sedation was conducted with sufentanil and midazolam during the procedure, which lasted over 4 hours.

The ablation procedures were done successfully without any complications.

\subsection{Case \#2}

A 65-year-old received a cardiac transplant due to end stage rheumatic valvular cardiomyopathy 13 years before the ablation procedure with a biatrial technique. He presented with refractory atrial flutter. His current medications included cyclosporine, azathioprine, prednisolone, diltiazem, atorvastatin, and furosemide. EP studies and ablation showed peri-mitral flutter. The procedure was performed under sedation with midazolam and fentanyl with an anesthetic time of 145 minutes. The procedure was done successfully, however the patient received 2 liters of normal saline as ablation catheter irrigation fluid and developed orthopnea, and pulmonary edema after the procedure which improved with intravenous furosemide.

\subsection{Case \#3}

A 49-year-old man received his heart transplant 24 years ago due to idiopathic cardiomyopathy by the biatrial technique. He developed paroxysmal rapid atrial flutter and underwent multiple cardioversions with medical treatment during the last 2 years, however his atrial flutter was refractory to those treatments and required an ablation procedure. His post transplant course was complicated by progressive renal failure and hypertension.

The EP study and pulmonary vein ablation were done using the transseptal approach under Transesophageal Echocardiography (TEE) which was technically very difficult. The patient received general anesthesia with an endotracheal tube, invasive intra-arterial monitoring and maintaining the activated clotting time $(\mathrm{ACT})>300$. As the procedure concluded, and the lines were taken out, the AV node was injured, and complete heart block occurred. The patient did not respond to atropine and had to be paced. The anaesthetic duration was over 6 hours.

\section{Discussion}

Cardiac arrhythmias are frequently encountered after heart transplantation. Previous studies demonstrated that about a quarter of these patients developed SVT attributed to macro-reentrant tachycardia, and about $4 \%$ underwent EP studies [2,3]. Anesthesia for ablation procedures in these patients mandate several considerations including transplant technique used, interventional cardiology procedure performed, and particular complications common to post transplant patients.

All three patients described had biatrial anastomosis which altered heart anatomy and predisposed the atrium to various re-entrant arrhythmias [7]. The third patient who received heart transplant for the longest time in the series (24 years before the ablation procedure) encountered a lot of technical difficulty during the lengthy procedure which is probably due to a combination of cardiac fibrosis and complicated anatomy.

The specific considerations for patients undergoing interventional cardiology procedures include pain management and increased incidence of complications. Pain caused by the procedure is of variable intensity due to radiofrequency ablation that generates heat and causes tissue damage [8]. An 8-mm-tip ablation catheter is generally used at our institution, power (20 - 50 Watt) and temperature use is limited to $50^{\circ} \mathrm{C}$, however power up to 100 Watt and temperature up to $65^{\circ} \mathrm{C}$ may be required. Post heart transplant patients, may have minimal pain due to neuronal denervation. Periprocedural complications are usually related to mechanical trauma or the use of radiofrequency power. This includes pericardial effusion, cardiac tamponade, thromboembolism, persistent defect of the inter atrial septum, aortic perforation, atrial perforation, atrio-esophageal fistula, acute coronary artery occlusion (circumflex artery), phrenic nerve injury, air emboli, periesophageal vagal injury, catheter entrapment in the mitral valve, dysrrhythmia, and complete heart block. An atrio-esophageal fistula is a rare but potentially fatal complication, and may manifest weeks after the ablation procedure [9]. If trans septal puncture is expected, TEE may enhance safety by allowing monitoring of catheter positioning as well as evaluation of complications [10,11]. Anticoagulant and ACT monitoring is crucial. Esophageal temperature monitoring may help reduce thermal injury from ablation [9].

Cardiac denervation causes impaired autonomic regulatory mechanism, delayed stress response, loss of vagal tone resulting in a resting heart rate of 90 - 100 per minute and alterations to demands for increased cardiac output. Therefore, maintenance of adequate preload is crucial. Coronary atherosclerosis will be accelerated (silent ischemia is likely). Renervation of the transplanted heart may occur, but to an unknown extent. The first patient, who underwent transplant 6 years before, had the atrial fibrillation originating from the receipient's atrial cuff which demonstrated the occurrence of renervation.

Immunosuppression is usually maintained with the three-drug regimen which includes cyclosporine, azathioprine and corticosteroids is common (tacrolimus and mycophenolate mofetil may replace cyclosporine and azathioprine, respectively). Frequent consequences of immunosuppressants include hypertension and renal impairment, which may affect choices of anesthetic agents, 
and are associated with tacrolimus and cyclosporine. Steroids can commonly cause hyperlipidemia and osteoporosis whereas Azathioprine frequently causes bone marrow suppression. Other infrequent complications include malignancy, gastrointestinal biliary disease, pancreatitis and peptic ulcer disease [10].

Drugs that act indirectly on the heart via either sympathetic or parasympathetic nervous system (ephedrine, atropine) will be ineffective. Drugs with a mixture of direct and indirect effects (digoxin, norepinephrine, and neostigmine) will exhibit only their direct effects. Agents with direct cardiac effects (epinephrine, isoproterenol) are more reliable.

Providing anesthesia for a high risk patient in a hostile environment outside of the operating room mandates careful assessment of the patient and meticulous arrangements for any possible complication and emergencies. Preoperative assessment should focus on graft function and end organs function, especially those that might be compromised by the long standing immunosuppressive therapy and possible dysfunction of the transplanted heart. Ablation procedures in a transplanted heart are technically challenging due to altered heart anatomy, intra thoracic position, and cardiac fibrosis which may increase the incidence of life threatening complications. Transcutaneous pacing and a defibrillator should be prepared as well as emergency resuscitation measures.

Procedures performed on the right side of the heart are usually less complicated than left sided procedures, which mandate inter-atrial septal puncture. For ablation within the right side of the heart, conscious sedation with adequate analgesia is adequate to ensure calm and pain free status without compromising the airway. However the expected long duration of the procedure in these patients may influence the anaesthetic choice. When deeper level of anesthesia or a prolonged procedure is required, laryngeal mask airway or intratracheal tube may be used. If TEE is required, deeper general anesthesia with secured airway with an endotracheal tube is mandatory.

In conclusion, the management of heart transplanted patients undergoing atrial ablation procedures includes a thorough history and physical examination, understanding of the procedure type, the transplanted heart anatomy and the heart and end organs function. Furthermore, special considerations should be addressed for management of high risk patients outside of the operating room in a remote location, lengthy procedures associated with technical difficulties, management of possible complications, and considerations in altered medications pharmacology. The anaesthetic plan should be tailored individually, and in collaboration with the cardiologists in order to provide the appropriate anaesthetic technique for the specific patient according to the planed intervention.

\section{REFERENCES}

[1] M. S. Mulligan, T. H. Shearon, D. Weill, F. D. Pagani, J. Moore and S. Murray, "Heart and Lung Transplantation in the United States, 1997-2006," American Journal of Transplantation, Vol. 8, No. 4p2, 2008, pp. 977-987. http://dx.doi.org/10.1111/j.1600-6143.2008.02175.x

[2] M. Vaseghi, N. G. Boyle, R. Kedia, J. K. Patel, D. A. Cesario, I. Wiener, J. A. Kobashigawa and K. Shivkumar, "Supraventricular Tachycardia After Orthotopic Cardiac Transplantation," Journal of the American College of Cardiology, Vol. 51, No. 23, 2008, pp. 2241-2249. http://dx.doi.org/10.1016/j.jacc.2008.02.065

[3] S. A. L. Ahmari, T. J. Bunch, A. Chandra, V. Chandra, K. Ujino, R. C. Daly, S. S. Kushwaha, B. S. Edwards, Y. F. Maalouf, J. B. Seward, C. G. McGregor and K. Chandrasekaran, "Prevalence, Pathophysiology, and Clinical Significance of Post-Heart Transplant Atrial Fibrillation and Atrial Flutter," The Journal of Heart and Lung Transplantation, Vol. 25, No. 1, 2006, pp. 53-60. http://dx.doi.org/10.1016/j.healun.2005.07.017

[4] H. Oral, C. Scharf, A. Chugh, B. Hall, P. Cheung, E. Good, S. Veerareddy, F. Pelosi and F. Morady, "Catheter Ablation for Paroxysmal Atrial Fibrillation: Segmental Pulmonary Vein Ostial Ablation versus Left Atrial Ablation,” 2003, pp. 2355-2360.

[5] F. Rodríguez-Entem, V. Expósito, S. González-Enríquez, T. García-Camarero and J. Olalla, "Atrial Flutter after Heart Transplantation: Mechanism and Catheter Ablation,” Transplantation Proceedings, Vol. 42, No. 7, 2010, pp. 2697-2701.

http://dx.doi.org/10.1016/j.transproceed.2010.04.067

[6] J. A. Mariani, M. A. McDonald, K. Nanthakumar, J. D. Parker and H. J. Ross, "Cardiac Resynchronization Therapy after Atrioventricular Node Ablation for Rapid Atrial Fibrillation in a Heart Transplant Recipient with Late Allograft Dysfunction," The Journal of Heart and Lung Transplantation, Vol. 29, No. 6, 2010, pp. 704-706. http://dx.doi.org/10.1016/j.healun.2010.01.009

[7] A. Jeffrey and N. M. E. Morgan, "Orthotopic Cardiac Transplantation: Comparison of Outcome Using Biatrial, Bicaval, and Total Techniques,” Journal of Cardiac Surgery, Vol. 20, No. 1, 2005, pp. 102-106. http://dx.doi.org/10.1111/j.0886-0440.2005.05011.x

[8] P. Lena, C. Mariottini, N. Balarac, J. Jacques Arnulf, A. Mihoubi and R. Martin, "Remifentanil versus Propofol for Radio Frequency Treatment of Atrial Flutter," Canadian Journal of Anesthesia, Vol. 53, No. 4, 2006, pp. 357362. http://dx.doi.org/10.1007/BF03022499

[9] A. Natale, A. Raviele, T. Arentz, H. Calkins, S. Chen, M. Haïssaguerre, G. Hindricks, Y. Ho, K. Kuck, F. Marchlinski, C. Napolitano, D. Packer, C. Pappone, E. Prystowsky, R. Schilling, D. Shah, S. Themistoclakis and A. Verma, "Venice Chart International Consensus Document on Atrial Fibrillation Ablation,” Journal of Cardiovascular Electrophysiology, Vol. 18, No. 5, 2007, pp. 560-580. http://dx.doi.org/10.1111/j.1540-8167.2007.00816.x

[10] L. Blier, M. Gilbert, J. Villeneuve, D. Mohty and G. O’hara, "Endothelial Progenitor Cells Restore Renal Function in Chronic Experimental Renovascular Disease," 
Pacing and Clinical Electrophysiology, Vol. 30, 2007, pp. 116-119.

[11] G. B. Chierchia, L. Capulzini, C. de Asmundis, A. Sarkozy, M. Roos, G. Paparella, T. Boussy, G. Van Camp, D.
Kerkhove and P. Brugada, "First Experience with RealTime Three-Dimensional Transoesophageal Echocardiography-Guided Transseptal in Patients Undergoing Atrial Fibrillation Ablation,” 2008, pp. 1325-1328. 